Sādhanā, Vol. 14, Part 3, December 1989, pp. 213-218. (C) Printed in India.

\title{
Maximum compatible classes from compatibility matrices
}

\author{
NRIPENDRA N BISWAS \\ Department of Electrical Communication Engineering, Indian Institute \\ of Science, Bangalore 560012, India
}

MS received 24 January 1990; revised 26 February 1990

\begin{abstract}
A fast algorithm for the computation of maximum compatible classes (MCC) among the internal states of an incompletely specified sequential machine is presented in this paper. All the maximum compatible classes are determined by processing compatibility matrices of progressingly diminishing order, whose total number does not exceed $(p+m)$, where $p$ is the largest cardinality among these classes, and $m$ is the number of such classes. Consequently the algorithm is specially suitable for the state minimization of very large sequential machines as encountered in VLSI circuits and systems.
\end{abstract}

Keywords. Compatibility; maximum compatible classes; incompletely specified sequential machines.

\section{Introduction}

Most algorithms for the state minimization of incompletely specified sequential machines (ISSM) require at some stage the set of maximum compatible classes (MCC). The extensively used method of determining the MCC for a given sequential machine (Kohavi 1978; Hill \& Peterson 1981; McCluskey 1986) consists of two steps. First, the compatible pairs (CP) of the machine are found by the implication chart of Paull \& Unger (1959). Second, the maximum compatible classes are computed by an exhaustive comparison among the compatible pairs. Since both the steps depend on exhaustive comparison, the complexity of the first step has the order $O\left(n^{2}\right)$, where $n$ is the number of states of the ISSM, and that of the second step has the order $O\left(m^{2}\right)$, where $m$ is the number of CP. Thus the overall complexity of the algorithm has the order $O\left(n^{4}\right)$. Hence this method is suitable only for small and medium size machines not exceeding, say, 15 states. However, with the advent of very large scale integration (VLSI), many hardware and software systems are now being designed as a collection of finite state sequential machines (DeMicheli \& Sangiovanni-Vincentelli 1983). Such machines are invariably incompletely specified and have very large number of internal states often exceeding 15 or 20 . In such a situation the existing algorithms are very uneconomical, both time and computation-wise. In this paper a new algorithm is presented for the fast computation of maximum compatible classes from the compatibility matrices with far less computation and time. The algorithm is also programable and therefore can be very conveniently developed into a computer-aided design (CAD) package for use in the design of VLSI circuits and systems. 


\section{The algorithm}

In describing the algorithm, the frequently used terms, such as, incompletely specified sequential machine (ISSM), compatibility, compatible pair (CP) and maximum compatible class (MCC) will be assumed to have their usual definitions as given in books on switching theory and logical design (Kohavi 1978; Hill \& Peterson 1981). We shall develop the algorithm by working out an example. Like other algorithms here also the compatibility relations between all pairs of states are determined by the implication chart. This compatibility relation is then depicted in the form of an $n \times n$ matrix where $n$ is the number of states of the ISSM. The rows and columns of the matrix are the states of the machine. A $1(0)$ is written at the intersection of a row and column when the corresponding pairs of states is compatible (incompatible). Table 1 shows the compatibility matrix $\mathrm{CM}_{1}$ of a 9-state ISSM showing the compatibility relations between all pairs as obtained from the implication chart. Note that the compatibility matrix $\mathrm{CM}_{1}$ is a symmetric matrix with an all-1 leading diagonal. This is so because each state is compatible with itself. In table 1 two more columns are added to $\mathrm{CM}_{1}$. The two additional columns give the weight $(\alpha)$ and candidate maximum compatible class (CMCC) of each row, which are defined below.

\section{DEFINITION 1}

The number of ones in a row will be called its weight.

It will be designated by $\alpha$.

Again each row of the other additional column gives the collection of all states which are compatible with the state heading the row. Thus in table 1 each of the states among $A B C D H I$ is compatible with state $A$, and each of the states BCDEH is compatible with the state $\mathrm{E}$.

\section{DEFINITION 2}

The collection of states which are compatible with a state will be called a candidate maximum compatible class (CMCC). The state with which each of the states of a CMCC is compatible will be called the generating state of the CMCC.

Thus every state of the machine, that is, every row of the $\mathrm{CM}_{1}$ generates a CMCc. It is also obvious that each MCC is either a CMCC itself or a subset of a CMCC. The algorithm finds all the maximum compatible classes of the ISSM from these candidate classes with the help of the following theorems and corollaries.

Table 1. Compatibility matrix $\left(\mathrm{CM}_{1}\right)$.

\begin{tabular}{llllllllllll}
\hline & A & B & C & D & E & F & G & H & I & $\alpha$ & CMCC \\
\hline A & 1 & 1 & 1 & 1 & 0 & 0 & 0 & 1 & 1 & 6 & ABCDHI \\
B & 1 & 1 & 1 & 1 & 1 & 0 & 1 & 1 & 1 & 8 & ABCDEGHI \\
C & 1 & 1 & 1 & 0 & 1 & 0 & 1 & 1 & 0 & 6 & ABCEGH \\
D & 1 & 1 & 0 & 1 & 1 & 0 & 0 & 1 & 1 & 6 & ABDEHI \\
E & 0 & 1 & 1 & 1 & 1 & 0 & 0 & 1 & 0 & 5 & BCDEH \\
*F & 0 & 0 & 0 & 0 & 0 & 1 & 1 & 0 & 0 & 2 & FG \\
G & 0 & 1 & 1 & 0 & 0 & 1 & 1 & 0 & 0 & 4 & BCFG \\
H & 1 & 1 & 1 & 1 & 1 & 0 & 0 & 1 & 1 & 7 & ABCDEHI \\
I & 1 & 1 & 0 & 1 & 0 & 0 & 0 & 1 & 1 & 5 & ABDHI \\
\hline
\end{tabular}


Theorem 1. A candidate maximum compatible class is a maximum compatible class if and only if it is one of the least cardinality CMCC among all the CMCCs and all its constituent states are pairwise compatible.

Proof. If (Sufficiency): If all the constituent states of a CMCC are pairwise compatible then the CMCC is an MCC if it is not contained in a larger MCC. Now, since the CMCC has the least cardinality, the generating state of the смCC has all its compatible states included in the CMCC and therefore there can be no MCC with greater cardinality which may contain the generating state within it. Therefore the CMCC cannot be contained in another larger MCC. Hence the CMCC is an MCC.

ONLY IF (Necessity): It is obvious that the two conditions which are sufficient are also necessary to make a CMCC an MCC.

QED

Note that all the elements of the compatibility matrix of an MCC are ones.

\section{COROLLARY. 1.1}

A CMCC of cardinality 1 or 2 is an MCC.

The proof of this corollary is obvious.

Theorem 2. A CMCC with the least cardinality which is itself not an $\mathrm{MCC}$ will generate more than one $\mathrm{MCC}$ with the generating state as one of its members. Each MCC produces an all-1 matrix, and its columns (or rows) form a subset of the CMCC which is not contained in a larger subset.

Proof. The CMCC with the least cardinality fails to become an MCC only when one (or more) pairs in it is (are) not compatible. Therefore, each of the subsets which produces an all-1 matrix has all its states pairwise compatible. Since the subset is not contained in a larger subset, it is an MCC.

QED

\section{COROLLARY. 2.1}

If there are two and only two zeros in the subcompatibility matrix of a СмCC, then the CMCC produces two maximum compatible classes.

Proof. If the element $a_{i i}$ of a subcompatibility matrix (SCM) is a 0 , then the element $a_{j i}$ will also be a 0 , since the SCM is a symmetric matrix. Now, this pair of zeros can be eliminated from the SCM in two ways, by deleting either the row and column $i$ or the row and column $j$. Hence, the SCM will produce two all-1 submatrices and, therefore, two MCCs.

QED

If the SCM of a $\mathrm{CMCC}$ has more than two zeros, then find all the MCC by following the generalized procedure as applied to a $\mathrm{CM}$.

Theorem 3. After all maximum compatible classes contained in a смсC have been determined, the generating state of the $\mathrm{CMCC}$ can be deleted from the rest of the candidate maximum compatible classes.

Proof. A смСC has all the states which are compatible with its generating state. 
Table 2. Compatibility matrix $\left(\mathrm{CM}_{2}\right)$

\begin{tabular}{ccccccccccc}
\hline & A & B & C & D & E & G & H & I & $\alpha$ & CMCC \\
\hline A & 1 & 1 & 1 & 1 & 0 & 0 & 1 & 1 & 6 & \\
B & 1 & 1 & 1 & 1 & 1 & 1 & 1 & 1 & 8 & \\
C & 1 & 1 & 1 & 0 & 1 & 1 & 1 & 0 & 6 & \\
D & 1 & 1 & 0 & 1 & 1 & 0 & 1 & 1 & 6 & \\
E & 0 & 1 & 1 & 1 & 1 & 0 & 1 & 0 & 5 & \\
${ }^{*}$ G & 0 & 1 & 1 & 0 & 0 & 1 & 0 & 0 & 3 & BCG \\
H & 1 & 1 & 1 & 1 & 1 & 0 & 1 & 1 & 7 & \\
I & 1 & 1 & 0 & 1 & 0 & 0 & 1 & 1 & 5 & \\
\hline
\end{tabular}

Therefore, all maximum compatible classes having the generating state in it are determined from the CMCC, and there cannot be any other MCC which may have the generating state in it. Hence the theorem.

QED

To apply theorem 1 to $\mathrm{CM}_{1}$ of table 1 , first the CMCC of the least cardinality is chosen. In this case it is the CMCC FG generated by the state F of the ISSM and it has a cardinality of 2 . By corollary $1.1 \mathrm{FG}$ can be listed as an MCC without any further processing. Once FG is selected as an MCC, the row and column of state F are deleted from $\mathrm{CM}_{1}$ as a direct consequence of theorem 3.

This is how $\mathrm{CM}_{2}$ (table 2) is derived from $\mathrm{CM}_{1}$.

Note that while $\mathrm{CM}_{1}$ was a matrix of order $9, \mathrm{CM}_{2}$ becomes a matrix of a reduced order, namely, 8. Again applying theorem 1 to $\mathrm{CM}_{2}$ the CMCC BCG generated by the state $G$ has the least cardinality. In order to check if the CMCC is an MCC, a submatrix with $\mathrm{B}, \mathrm{C}$ and $\mathrm{G}$ as rows and columns is derived from $\mathrm{CM}_{2}$. This is called the subcompatibility matrix $\mathrm{SCM}_{2}(\mathrm{BCG})$ and is shown in table 3 . Obviously all the states will be pairwise compatible, if the submatrix is an all-1 matrix. It can be seen that the submatrix $\mathrm{SCM}_{2}(\mathrm{BCG})$ is an all-1 matrix, as all the row weights are 3. Hence, by theorem 1, BCG is an MCC. After BCG is selected as an MCC, state G is deleted from the rows and columns of $\mathrm{CM}_{2}$, and $\mathrm{CM}_{3}$ as shown in table 4 is obtained.

Table 3. Subcompatibility matrix $\mathrm{SCM}_{2}$ (BCG).

\begin{tabular}{lllll}
\hline & B & C & G & $\alpha$ \\
\hline B & 1 & 1 & 1 & 3 \\
C & 1 & 1 & 1 & 3 \\
G & 1 & 1 & 1 & 3 \\
\hline
\end{tabular}

Table 4. Compatibility matrix $\left(\mathrm{CM}_{3}\right)$.

\begin{tabular}{cccccccccl}
\hline & A & B & C & D & E & H & I & $\alpha$ & CMCC \\
\hline A & 1 & 1 & 1 & 1 & 0 & 1 & 1 & 6 & \\
B & 1 & 1 & 1 & 1 & 1 & 1 & 1 & 7 & \\
${ }^{*}$ C & 1 & 1 & 1 & 0 & 1 & 1 & 0 & 5 & ABCEH \\
D & 1 & 1 & 0 & 1 & 1 & 1 & 1 & 6 & \\
${ }^{*}$ E & 0 & 1 & 1 & 1 & 1 & 1 & 0 & 5 & BCDEH \\
H & 1 & 1 & 1 & 1 & 1 & 1 & 1 & 7 & \\
$* 1$ & 1 & 1 & 0 & 1 & 0 & 1 & 1 & 5 & ABDHI \\
\hline
\end{tabular}


Table 5. Subcompatibility matrix (SCM) $)_{3}(\mathrm{ABCEH})$.

\begin{tabular}{ccccccc}
\hline & A & B & C & E & H & $\alpha$ \\
\hline A & 1 & 1 & 1 & 0 & 1 & 4 \\
B & 1 & 1 & 1 & 1 & 1 & 5 \\
C & 1 & 1 & 1 & 1 & 1 & 5 \\
E & 0 & 1 & 1 & 1 & 1 & 4 \\
H & 1 & 1 & 1 & 1 & 1 & 5 \\
\hline & & & ABCH & & &
\end{tabular}

In this table three candidate maximum compatible classes generated by the states $\mathrm{C}, \mathrm{E}$ and I have the least cardinality of 5 . The corresponding candidate classes are now processed one after another. First the submatrix $\operatorname{scm}_{3}(\mathrm{ABCEH})$ as shown in table 5 is derived. The row weights of the SCM are not 5 in all rows. It has two rows $A$ and $E$ having weights 4 . It can be easily seer that the submatrix of the $\mathbf{S C M}, \mathrm{SCM}_{3}$ $(\mathrm{ABCH})$ will be an all-1 matrix, as the row and column $\mathrm{E}$ having the 0 will be absent. Similarly the $\operatorname{SCM}_{3}(\mathrm{BCEH})$ will also be an all-1 matrix as the row and column $\mathrm{A}$ having the 0 will be absent. Hence, by Corollary $2.1 \mathrm{ABCH}$ and $\mathrm{BCEH}$ are maximum compatible classes. Note that before deriving the $\operatorname{SCM}_{3}(\mathrm{BCDEH})$ the state $\mathrm{C}$ is deleted from the $\mathrm{CMCC}$ as the $\mathrm{CMCC}$ of the generating state $\mathrm{C}$ have been processed (theorem 3). Hence, $\mathrm{SCM}_{3}(\mathrm{BDEH})$ (table 6) is derived from $\mathrm{CM}_{3}$. This is an all-1 matrix (as all the row weights are 4). Therefore BDEH is an MCC. After this the submatrix $\mathrm{SCM}_{3}(\mathrm{ABDHI})$ is derived. This produces the MCC ABDHI. This completes the processing of all the least cardinality candidate maximum compatible classes of $\mathrm{CM}_{3}$. Before deriving $\mathrm{CM}_{4}$ from $\mathrm{CM}_{3}$, the number of rows of $\mathrm{CM}_{4}$ should be calculated. Here it is 4 . Therefore, if $\mathrm{CM}_{\mathbf{4}}$ produces any $\mathbf{M C C}$, its cardinality must be less than those

Table 6. Subcompatibility matrix $\left(\mathrm{SCM}_{3}\right)(\mathrm{BDEH})$.

\begin{tabular}{llllll}
\hline & B & D & E & H & $\alpha$ \\
\hline B & 1 & 1 & 1 & 1 & 4 \\
D & 1 & 1 & 1 & 1 & 4 \\
E & 1 & 1 & 1 & 1 & 4 \\
H & 1 & 1 & 1 & 1 & 4 \\
\hline \multicolumn{5}{c}{ BDEH }
\end{tabular}

Table 7. Subcompatibility matrix $\left(\mathrm{SCM}_{3}\right)(\mathrm{ABDHI})$.

\begin{tabular}{ccccccc}
\hline & A & B & D & H & I & $\alpha$ \\
\hline A & 1 & 1 & 1 & 1 & 1 & 5 \\
B & 1 & 1 & 1 & 1 & 1 & 5 \\
D & 1 & 1 & 1 & 1 & 1 & 5 \\
H & 1 & 1 & 1 & 1 & 1 & 5 \\
I & 1 & 1 & 1 & 1 & 1 & 5 \\
\hline
\end{tabular}


computed from $\mathrm{CM}_{3}$. But all such maximum compatible classes must have already been determined. Hence, there is no need to derive or process $\mathbf{C M}_{\mathbf{4}}$. Procedure terminates here. All the MCCs of the machine are FG, BCG, ABCH, BCEH, BDEH

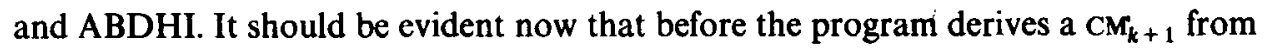
$\mathrm{CM}_{k}$, it must check if the number of remaining rows of $\mathrm{CM}_{k}$ is less than the cardinality of the $\mathrm{MCC}$ found in $\mathrm{CM}_{k}$. If yes, then the program produces a "stop derivation" signal which ends the algorithm.

The algorithm can now be summarized as follows.

Begin

DERIVE_COMPATIBILITY_MATRIX $\mathrm{CM}_{1}$ of the given machine FIND_MAXIMUM COMPATIBLE CLASSES of cardinality 1 and 2 from CM $_{1}$ DERIVE_NEXT_MATRIX and COMPUTE_MAXIMUM COMPATIBLE CLASSES bv deriving submatrices and checking for all-1 matrices

Repeat this procedure

Until STOP_DERIVATION signal is received

\section{End}

\section{Conclusion}

It is evident from the above description of the algorithm that to determine all the maximum compatible classes, the number of compatibility matrices which need to be derived from the previous matrices starting from $\mathrm{CM}_{1}$ will not exceed the largest cardinality among the maximum compatible classes, and the number of all-1 submatrices to be derived and processed will not exceed the total number of such classes. The processing of matrices also does not involve expensive matrix operations such as multiplication or inversion. For these reasons the algorithm becomes much faster than existing ones, specially for large and very large incompletely specified sequential machines. To compute all 30 MCC of a 22-state machine the compatibility matrix algorithm took only $0.755 \mathrm{~s}$ of CPU time in a DEC 1090 computer, compared to $5.329 \mathrm{~s}$ of CPU time (Chakraborty 1987) in the same computer by the existing algorithm as presented in Kohavi (1978), Hill \& Peterson (1981), and McCluskey (1986).

\section{References}

Chakraborty A 1987 On state minimization of very large incompletely specified sequencial machines, BE project report, Department of Electrical Communication Engineering, Indian Institute of Science, Bangalore

DeMicheli G, Sangiovanni-Vincentelli A 1983 Computer aided synthesis of PLA based finite state machines, Proc. Int. Conf. on Computer-Aided Design (Washington, DC: IEEE Comput. Soc. Press) pp. 154-156

Hill F J, Peterson G R 1981 Introduction to switching theory and logical design 3rd ed (New York: Wiley)

Kohavi Z 1978 Switching and finite automata theory, 2nd edn (New York: McGraw-Hill)

McCluskey E J 1986 Logic design principles (Engelwood Cliffs, NJ: Prentice-Hall)

Paull M C, Unger S H 1959 IRE Trans. Electron. Comput. EC-8: 356-357 